\title{
The practice of clinical genetics: A survey of practitioners
}

\author{
Beth A. Pletcher, MD, FAAP, FACMG ${ }^{1}$, Ethan A.B. Jewett, $M A^{2}$, William L. Cull, PhD ${ }^{3}$, Sarah E. Brotherton, PhD ${ }^{4}$, \\ H. Eugene Hoyme, MD, FAAP ${ }^{5}$, Richard J.D. Pan, MD, MPH, FAAP ${ }^{6}$, and Holly J. Mulvey, $M A^{7}$
}

Purpose: A survey of clinical geneticists was undertaken to learn more about current practice. Methods: An attempt
was made to survey all geneticists practicing in the United States to elicit information on a variety of issues.
Results: A total of $69 \%$ of geneticists responded. Most practice at a medical school, most receive a portion of their
income from salary, and many receive personal financial support from research grants. Conclusion: The specialty
of medical genetics is primarily housed in tertiary care settings. Although a substantial amount of time is required
to see patients, reimbursement for these services does not cover the costs to maintain such practices. Genet Med 2002:4(3):142-149.

Key Words: health care, workforce, survey, geneticists, clinicians

Over the past decade much has been written about the $\mathrm{Hu}-$ man Genome Project, informed consent, genetic predisposition testing, and integration of genetics into primary care practice, yet very little has been published describing the actual practice of medical genetics or the demand for genetic services. ${ }^{1-3}$ Those studies that have been conducted have provided useful information concerning the economics of clinical genetic services within a single academic center, ${ }^{1,2}$ and the experiences with predictive genetic testing of HMO directors and directors of academic genetic centers. ${ }^{3}$ Before this survey, however, no systematic study had been undertaken to gather practice information from all geneticists practicing in the United States.

The first certifying examination in clinical genetics was administered by the American Board of Medical Genetics (ABMG) in 1981, but the specialty of medical genetics was not recognized by the American Board of Medical Specialties until 1996, after the establishment of the American College of Medical Genetics. In their 1987 articles, Bernhardt et al. point out that as geneticists deal with a wide variety of relatively rare disorders, the practice may in fact overlap with any and all primary care and medical specialties or subspecialties. ${ }^{1,2}$ Furthermore, some geneticists practice as consultants only,

\footnotetext{
From the ${ }^{I}$ Department of Pediatrics, University of Medicine and Dentistry-New Jersey Medical School, Newark, New Jersey; ${ }^{2}$ Division of Graduate Medical Education and Pediatric Workforce, American Academy of Pediatrics, Elk Grove Village, Illinois; ${ }^{3}$ Division of Health Policy Research, American Academy of Pediatrics, Elk Grove Village, Illinois; ${ }^{4}$ Department of Data Acquisition Services, American Medical Association, Chicago, Illinois; ${ }^{5}$ Department of Pediatrics, Stanford University School of Medicine, Stanford, California; ${ }^{6}$ Department of Pediatrics, University of California Davis Medical Center, Sacramento, California; and ${ }^{7} \mathrm{Fu}$ ture of Pediatric Education II (FOPE II) Project, American Academy of Pediatrics, Elk Grove Village, Illinois.

Beth A. Pletcher, MD, Center for Human and Molecular Genetics, 90 Bergen Street, Suite 5400, Newark, NJ 07103-2499.

Received: December 12, 2001.

Accepted: February 1, 2002.
}

whereas others take on a more significant role as managers of complex genetic patient care. Due to the large cognitive component with emphasis on preventive health care provided in a primarily outpatient care setting, as well as several other economic factors, these authors contended that reimbursement for clinical genetic services is far less than is necessary to be self-supporting. They also noted that the average amount of time devoted to a single new patient visit was 7.1 hours and a follow-up visit was 4.0 hours. This compared rather unfavorably with the average collection for these services of $\$ 135.00$ ( $\$ 19.00 /$ hour) and $\$ 49.00$ (\$12.00/hour), respectively.

As a relatively new medical specialty, medical genetics is evolving to keep up with rapid and unprecedented technological advances, as well as changes in clinical practice driven by a competitive health care marketplace. Although there are many similarities between the specialty of genetics and certain pediatric subspecialties, there are also unique aspects of the practice of genetics that set it apart. To explore what the actual day-today practice of medical genetics entails more than a decade after the findings of Bernhardt et al. were published, physicians who currently practice genetics were surveyed by the Future of Pediatric Education II (FOPE II) Project on a variety of issues regarding their practice.

The FOPE II Project was a 3-year, grant-funded initiative of the entire pediatric community. As part of the project, key leaders in the pediatric community addressed the future supply and training of pediatricians and the provision of pediatric care in the new millennium. The results of their deliberations are encompassed in the final report of the FOPE II Task Force, "Organizing Pediatric Education to Meet the Needs of Infants, Children, Adolescents, and Young Adults in the 21st Century." ${ }^{4}$ An important component of the FOPE II Project was the gathering of insights, information, and data, which served as the underpinning of the Task Force recommendations. As part of this endeavor, 17 medical and surgical specialty and subspecialty sections of the American Academy of Pediatrics (AAP) 
and appropriate specialty societies participated in the FOPE II Survey of Sections Project.

\section{SUBJECTS AND METHODS}

\section{Survey overview}

Two surveys, the first focusing on general workforce topics and the second focusing specifically on clinical genetics, were conducted simultaneously to gather information from as many clinical geneticists as could be located in the United States (the surveys are available from the authors). The general workforce survey was developed for all 17 AAP sections that were surveyed as part of the FOPE II project. It queried respondents on demographics, specialty and subspecialty training, apportionment of time among professional activities, referral patterns, and sources of practice competition. The clinical genetics survey was designed to solicit information specific to the practice of clinical genetics and was developed by members from the AAP Section on Genetics and Birth Defects. Survey domains included board certification, practice characteristics, professional activities, income sources, patient profiles, research support, and the role of genetic counselors in group practice. Both surveys were pretested by a small group of 11 geneticists, and questionnaires were modified based on comments received. All responses to the surveys were kept confidential, and the study was reviewed by the Institutional Review Board of the American Academy of Pediatrics.

\section{Sample}

Because not all clinical geneticists are trained in the specialty of pediatrics, a decision was made to invite all geneticists who are board certified in clinical genetics to participate. A total of 903 physicians were identified as geneticists and contacted. This sample included all members of the AAP Section on Genetics and Birth Defects and all geneticists certified in clinical genetics by the ABMG. Each mailing to the sample contained the general workforce survey, the clinical genetics survey, a cover letter emphasizing the importance of the survey, and a return envelope. Up to five mailings of the survey were sent to participants between November 1997 and March 1998. Of the original sample of 903, 168 geneticists were excluded because they were no longer practicing in the United States or were still in training. Of the remaining 735 practicing geneticists, 506 $(69 \%)$ completed and returned the surveys.

We were able to obtain age and gender information for nonresponding AAP section members. The AAP section nonrespondents were significantly older than the AAP respondents ( 54.7 years vs. 50.5 years, $P=0.005$ ). Although females were more likely to respond, gender did not differ significantly between nonrespondents and respondents (35\% female vs. $44 \%$ female, $P=0.207$ ).

Descriptive statistics were calculated for all response variables. For individual questions, the total of 506 responses produced $95 \%$ confidence intervals of plus or minus a sampling error of $4.4 \% .^{5}$ The number of cases for each variable fluctu- ated slightly based on the number of missing values for the individual question.

\section{RESULTS}

\section{Demographics}

Fifty-nine percent of the respondents were male, and $41 \%$ were female. The average age of the respondents was 48 years. Ethnic distribution was as follows: $84 \%$ white/non-Hispanic, $6 \%$ Asian/Pacific Islander, $4 \%$ white/Hispanic, and $1 \%$ African American. The remaining 5\% identified themselves as belonging to other racial or ethnic groups. The majority $(84 \%)$ of respondents graduated from medical schools in the United States, whereas $2 \%$ graduated from Canadian medical schools, and the remaining $14 \%$ from other medical schools.

\section{Education and certification}

The average year of graduation from medical school for all respondents was 1977, which correlates well with the average age of 48 years. Almost $98 \%$ indicated that they had formal training in medical genetics, and over $89 \%$ were board certified in medical genetics. Other specialty training included pediatrics, obstetrics and gynecology, maternal-fetal medicine, internal medicine, as well as others (see Table 1). Most respondents had training in multiple specialties, which explains why the percentages in Table 1 add up to more than $100 \%$. In addition to receiving board certification in clinical genetics, $6.5 \%$ of respondents indicated that they also received board certification in clinical biochemical genetics, $6 \%$ in clinical cytogenetics, and $3.4 \%$ in clinical molecular genetics.

In describing their genetic fellowship or residency, geneticists indicated that approximately $50 \%$ of their time was spent in direct patient care, $23 \%$ in basic science research, $13 \%$ in a clinical genetic laboratory, and $13 \%$ in clinical research. However, fellowship experiences varied widely, and $30 \%$ of respondents stated they spent no time doing clinical research, and $44 \%$ spent no time in basic science research. When asked about the training areas deserving more attention, clinical biochemical genetics (46\%), administration (46\%), laboratory biochemical genetics (45\%), clinical research skills (39\%), and basic science research skills (36\%) were reported most often.

Table 1

Specialty training and board certification

\begin{tabular}{lccccc}
\hline & \multicolumn{2}{c}{ Training } & & \multicolumn{2}{c}{ Board certification } \\
\cline { 2 - 3 } Specialty & No. & $\begin{array}{c}\text { \% of } \\
\text { total }\end{array}$ & & No. & $\begin{array}{c}\% \text { of } \\
\text { subgroup }\end{array}$ \\
\hline Medical genetics & 495 & 98 & & 442 & 89 \\
General pediatrics & 382 & 76 & & 346 & 91 \\
Obstetrics and gynecology & 41 & 8 & & 35 & 85 \\
Maternal-fetal medicine & 27 & 5 & & 16 & 59 \\
Internal medicine & 26 & 5 & & 20 & 77 \\
Other specialties & 54 & 11 & & 35 & 65 \\
\hline
\end{tabular}


On the other hand, $7 \%$ of the fellowship trained individuals believed that their training program should have devoted less time to basic science research skills, and this was the area most frequently targeted for reduced time.

\section{Practice location}

Geographic data collected shows that $47 \%$ of respondents practice in an urban, non-inner city setting, 34\% in an urban, inner city setting, $15 \%$ in a suburban setting, and only $4 \%$ in a rural setting. When asked more specifically for the size of the populations served, approximately $60 \%$ of respondents reported practicing in large metropolitan areas with a population of $>1,000,000,16 \%$ reported practicing in an area with a population of 500,000 to $1,000,000,19 \%$ reported practicing in an area with a population of 100,000 to 499,999 , whereas only $5 \%$ reported practicing in an area with $<100,000$ residents. Respondents indicated that on average there are eight clinical geneticists serving their catchment area.

Almost two thirds of respondents indicated that their primary practice site was located at a medical school. Additionally, $6.4 \%$ practice in a community hospital, $5.3 \%$ practice within a specialty group, $4.5 \%$ work for a health maintenance organization, and $4.3 \%$ work within a multispecialty group. Only $2.7 \%$ indicated that they are in a pediatric group practice, another $2.7 \%$ are in solo practice, $1.2 \%$ work in a uniformed health services clinic, and $1 \%$ are part of a community health center.

\section{Referral patterns}

Over $90 \%$ of respondents care for pediatric genetic patients. As shown in Table 2, referrals to geneticists come from many different sources. The most common sources reported included referrals from pediatricians (95\%), family physicians $(87 \%)$, pediatric medical or surgical subspecialists (86\%), and obstetrician-gynecologists (78\%). When asked about changes in the volume of referrals over the previous practice year, $36 \%$ noted an increase in volume, $12 \%$ noted a decrease, and 52\% reported no change. Of those who reported an increase in the

Table 2

Sources of referrals for geneticists

\begin{tabular}{lc}
\hline Referral source & $\begin{array}{c}\text { Percentage reporting } \\
\text { any referrals } \\
\text { from source }\end{array}$ \\
\hline Pediatric generalists & 95 \\
Family physicians & 87 \\
Pediatric subspecialists & 85 \\
Obstetricians/gynecologists & 78 \\
General internists & 60 \\
Adult medicine subspecialists & 58 \\
Pediatric nurse practitioners & 57 \\
Physician assistants & 34 \\
Others & 24 \\
\hline
\end{tabular}

volume of referrals, $61 \%$ reported increasing numbers of referrals from adult subspecialists. Of this same group who noted an increase in volume, 39\% believed that there was a trend for general pediatricians and other generalists not to treat the more complex genetic patients themselves. When the complexity of referrals was examined for all respondents, $34 \%$ reported an increase in the complexity of referrals, only $1 \%$ reported a decrease in referral complexity, and $65 \%$ reported no change.

\section{Competitive forces}

Half of respondents reported competition for pediatric genetic patients in their geographical regions. Competition was perceived most frequently by geneticists practicing in an urban, inner city $(57 \%)$ or suburban location $(63 \%)$ and less frequently by those in an urban, non-inner city (43\%) or rural setting $(33 \%)\left(\chi^{2}=13.7, P=0.003\right)$. For those who faced competition, $87 \%$ indicated that a major source of competition was pediatric subspecialists, $19 \%$ reported substantial competition from pediatricians, 9.5\% reported competition from family physicians, and 6\% reported competition from nonphysician personnel.

Approximately $35 \%$ of geneticists modified their practices in response to these competitive forces. Of this subgroup, $40 \%$ increased their office hours, $32 \%$ decreased their research and administrative activities, $21 \%$ increased their research and administrative activities, $20 \%$ decreased their support staff, and $18 \%$ decreased their fees.

Whether respondents believed there was a need for additional pediatric subspecialists or geneticists in their communities over the next 3 to 5 years was significantly associated to whether respondents faced competition $\left(\chi^{2}=10.8, P<\right.$ 0.001 ). Of the respondents who reported facing competition, only $38 \%$ indicated that there would be a need for additional subspecialists or geneticists in their community; whereas $53 \%$ of those not facing competition indicated additional need in their community.

\section{Sources of income}

Although the survey did not ask respondents to disclose their salaries and other earnings, it did query them about the sources of their income. Most medical geneticists (83\%) receive some or all of their income from salary only or from salary plus performance incentives. A total of $30 \%$ of respondents also receive some income from fee for service, and $26 \%$ receive income in part from prepaid capitated sources. Almost three quarters of respondents indicated that most (67-100\%) of their income came from straight salary or salary plus incentive, $20 \%$ received the majority of their salary through a prepaid capitated arrangement, and only $6 \%$ from traditional fee for service.

\section{Practice overview}

Geneticists reported working an average of 57 hours per week. Table 3 shows the mean percentages of time devoted to various practice activities. More than half of this time (54\%) is 
Table 3

How geneticists divide their time

\begin{tabular}{lc}
\hline Professional activity & $\begin{array}{c}\text { Percentage } \\
\text { of time } \\
\text { (mean) }\end{array}$ \\
\hline Direct patient care & 30 \\
Outpatient setting & 11 \\
Multispecialty clinics & 8 \\
Inpatient setting & 5 \\
Other clinical activity & 13 \\
Nonpatient care & 12 \\
Administration & 11 \\
Basic science research & 9 \\
Teaching & 1 \\
Clinical or health services research & 9 \\
Other, nondirect patient care & \\
\hline
\end{tabular}

devoted to direct patient care activities, and most of this is provided in genetic or pediatric outpatient settings. Of the time that is not devoted to direct patient care, there is a fairly equal balance between administration, basic science research, teaching, and clinical or health services research.

Approximately $74 \%$ of respondents are actively involved in one or more specialty clinics, and of those, $70 \%$ indicated that they serve as a director in at least one of these clinics, whereas $62 \%$ serve as the team geneticist, but not as a director in at least one specialty clinic. For those specialty clinics directed by the geneticist, $42 \%$ of these are medical specialty clinics, $29 \%$ single gene disorder clinics, $12 \%$ surgical specialty clinics, and the remaining $17 \%$ other types of clinics. For those specialty clinics where the responding geneticist serves as a team member only, $35 \%$ of those are surgical specialty clinics, 30\% medical specialty clinics, $22 \%$ single gene disorder clinics, and $12 \%$ other types of clinics.

Forty-three percent of respondents indicated they serve as inpatient primary care attendings, although only $28 \%$ actually care for primary care inpatients. This discrepancy may reflect the newer role of inpatient teaching attending versus service attending, and the increasing role of hospitalists in many large medical centers. Of those geneticists serving as inpatient primary care attendings, over $60 \%$ were on the pediatric service, $8 \%$ on the obstetric and gynecologic service, whereas $18 \%$ served on other specialty services. These primary care attendings spend an average of 9.5 weeks per year on service, with a median of 4 weeks.

On average, geneticists practice in groups composed of three geneticists and three genetic counselors. Clearly, genetic counselors are an integral part of the practice of genetics, with respondents identifying a multitude of functions that are performed by counselors. Of respondents, $92 \%$ indicated that genetic counselors in their group took pedigrees on outpatients, $84 \%$ indicated that counselors saw outpatients in col- laboration with a geneticist, $82 \%$ indicated that counselors wrote summary letters to patients, $55 \%$ indicated that counselors wrote or composed clinic notes, $50 \%$ indicated that counselors saw inpatients in collaboration with a geneticist, 50\% indicated that counselors saw outpatients independently, and $19 \%$ indicated that counselors saw inpatients independently.

\section{Clinical genetic patients}

Breakdown of patients seen by geneticists by percentage of practice indicates that prenatal patients and infants or young children represent the greatest patient volume (see Table 4). Approximately $25 \%$ of respondents do not provide any care to prenatal patients, and 5\% provide care exclusively to prenatal patients. Approximately $13 \%$ do not provide care to newborns, and $19 \%$ do not provide care to non-prenatal adolescents or adults.

Insurance coverage for genetic patients seen by respondents varied widely. On average, $35 \%$ of patients had straight Medicaid or SSI, $34 \%$ had HMO/PPO/IPA coverage, $18 \%$ had traditional health insurance, $8 \%$ were self-pay, and $5 \%$ had other forms of insurance or payment arrangements.

On average, each geneticist sees seven new and six follow-up patients per week, and two thirds of these individuals indicated they could reasonably see more genetic patients than they are seeing at present. Respondents spend, on average, 3.1 hours with new patients, including library, research, and letter writing time, whereas a follow-up visit requires on average 1.4 hours. Table 5 outlines the estimated time needed by geneticists to serve new and follow-up patients.

\section{Geneticists and research}

Thirty-eight percent of respondents receive outside funding for research, with the average annual amount of funding of $\$ 199,000$ (median $\$ 126,000$ ) for direct costs, and $\$ 97,000$ (median $\$ 50,000)$ for indirect costs. Of those receiving funding, $68 \%$ receive personal salary support from these research grants, and the geneticists in this subset dedicate approximately half of their professional time to this research.

\section{Retirement}

The average age of respondents was 48 , and overall they planned to retire at age 66 . Female geneticists were on average

\section{Table 4}

Age breakdown of genetic patients

\begin{tabular}{lc}
\hline Age group (years) & $\begin{array}{c}\% \text { of } \\
\text { patients }\end{array}$ \\
\hline Prenatal & 21 \\
Newborns & 18 \\
Toddlers (1-2) & 18 \\
Preschool (3-4) & 13 \\
Middle childhood (5-11) & 10 \\
Non-prenatal adolescents (12-17) & 7 \\
Non-prenatal adults (18 and older) & 12 \\
\hline
\end{tabular}


Table 5

Average total time spent per patient: percentage of respondents checking each time range

\begin{tabular}{lcc}
\hline $\begin{array}{l}\text { Time } \\
\text { (in hours) }\end{array}$ & $\begin{array}{c}\text { New } \\
\text { patient } \\
(\%)\end{array}$ & $\begin{array}{c}\text { Follow-up } \\
\text { patient } \\
(\%)\end{array}$ \\
\hline$\leq 0.5$ & 0 & 25 \\
$0.5-1$ & 13 & 37 \\
$1-2$ & 36 & 30 \\
$2-3$ & 26 & 8 \\
$3-4$ & 14 & 0 \\
$>4$ & 11 & 0 \\
\hline
\end{tabular}

4 years younger than their male counterparts and planned to retire 2 years earlier. Only $10 \%$ of respondents intended to retire in the next 5 years.

\section{DISCUSSION}

In 1987, a group of geneticists and genetic counselors set out to review the evolution of the specialty of medical genetics and to evaluate the time spent and costs of providing genetic services to patients in an urban, academic setting. ${ }^{1,2}$ These authors found that genetic services required a substantial professional time commitment and were poorly reimbursed. The present study confirms these previous findings and provides much needed data on the unique aspects of genetic practice.

The FOPE II survey data indicate that many characteristics of genetic practice have not changed in the past 14 years. The vast majority of geneticists identify themselves as white/nonHispanic, whereas Hispanic and African American practitioners are grossly underrepresented. As the United States continues to undergo ethnocultural changes and population redistribution, it is imperative that the racial and ethnic diversity of the genetics workforce be enhanced in the interest of optimal patient care. Indeed, prior research has demonstrated the influence of racial, ethnic, linguistic, cultural, and other concordances between the physician and patient on health outcomes. ${ }^{6-9}$ The FOPE II data also underscore the importance of including training in the multicultural dimensions of health care as part of genetic residency training programs.

Unlike most pediatric subspecialties, in which practitioners are trained exclusively in the specialty of pediatrics, geneticists are a very diverse group of physicians. Although our survey clearly shows that the vast majority of practicing geneticists have prior training in pediatrics, increasing numbers of physicians from a variety of medical and surgical specialties are entering the field. This trend reflects the fact that genetic disorders may be encountered in patients of all ages, with a wide range of organ system involvement.

Fellowship training in genetics was generally well regarded by the survey respondents, although many expressed a great interest in receiving more training in clinical biochemical genetics and administrative skills. Some respondents would also have liked more training in both clinical and basic science research, an interest probably driven by the substantial amount of time many practicing geneticists spend in research as well as the promotion criteria in academic settings in which most geneticists work. In fact, the need for strong research training is supported by the survey data that suggest that the clinical geneticist's income is often supplemented by research dollars in a tertiary-care practice setting.

Clinical geneticists $(66 \%)$ primarily practice in a medical school setting, which is more than other pediatric subspecialties surveyed, except pediatric infectious diseases (ID) (70\%). ${ }^{10}$ The majority of clinical geneticists likewise tend to practice in a market with population bases greater than one million. Merely $2 \%$ of geneticists function in a solo practice setting, with only critical care (2\%) and emergency medicine (EM) $(0 \%)$ at or below this level. ${ }^{10}$ Only $14 \%$ of geneticists find themselves in a freestanding group practice, which is less than all subspecialties, except for ID (12\%) and EM (11\%). ${ }^{10}$ The location of geneticists in academic medical centers may be influenced by many factors, including their dependence on research funding to supplement income and a reliance on internal and external providers for patient referrals and collaborative care of complex patients. These and other factors may serve as barriers to geneticists who wish to establish themselves in solo practice.

Slightly over half of the geneticist's work time is devoted to direct patient care, which is less than all pediatric subspecialties, except ID, for which only $42 \%$ of work time for these specialists is in direct patient care..$^{10}$ In the year preceding the survey, many more geneticists had noted an increase in the volume and/or complexity of referrals, which is similar to other pediatric subspecialty practices..$^{10}$ One potential reason for increasing complexity of referrals is that primary care physicians may feel increasing pressure to follow less complex cases themselves (i.e., a child or adult with Down syndrome, Fragile X syndrome, or neurofibromatosis) and to refer only the most medically complex cases to the genetic specialist. Rapid advances in the field of genetics are also increasing the complexity of care for practicing geneticists. Although $50 \%$ of geneticists experienced competition for patients from outside sources, this rate is lower than that reported for other pediatric subspecialties, with the exception of ID. ${ }^{10}$ It is possible that geneticists and ID specialists perceive less competition, because of their medical school milieu and fewer number of direct patient care hours. In addition, referring specialists may not feel as comfortable managing or diagnosing these types of patients. Overall, geneticists and pediatric subspecialists as a whole believed that their greatest source of competition was from other pediatric subspecialists. ${ }^{10}$

In addition to caring for genetic patients, many clinical geneticists serve as inpatient primary care attendings. Furthermore, more than $75 \%$ of geneticists serve as the director and/or work as the team geneticist in a multidisciplinary specialty clinic; these practice opportunities may be more available in tertiary care setting or relatively large catchment areas. Most geneticists, even with prior pediatric training, devote substantial 
time to caring for prenatal and nonprenatal adult patients with genetic concerns. As more individuals enter the field of genetics from nonpediatric specialties, it is likely that there will be increasing subdivision of genetic care. Subdivision of care might dictate that pediatric geneticists primarily see infants, children, and adolescents for genetic services; obstetric or maternal-fetal trained geneticists care for pregnant women or couples to address prenatal issues; and internists with genetic training care for adult patients who have concerns about cancer risk assessment, diagnosis, or management of adult onset diseases.

As patients with genetic disorders grow older, the health care system will need to be restructured to allow patients to make a smooth transition from pediatric to adult medical specialist care. Although the Society of Teachers of Family Medicine has taken the initiative to develop a comprehensive, family-medicine oriented genetic curriculum, it is not clear how other specialties and societies are addressing these needs, if at all. It has been suggested that, in light of the paucity of trained clinical geneticists, internists will shoulder the primary responsibility in the future for providing genetic counseling, including helping patients to understand genetic testing and therapy. In this model, the internist would have a consultative arrangement with a clinical geneticist and would use the genetic professional for patient information but not referral. ${ }^{11}$

In this scenario, the clinical geneticists would serve as educators to internists and others providing genetic care. Indeed, the FOPE II data reveal that respondents are already spending over $10 \%$ of their time teaching and lecturing to both trainees and attending physicians. As educational forums expand, including online resources, it is possible that more and more primary care providers will look to geneticists to keep them abreast of new genetic breakthroughs and diagnostic testing. With extensive provider education by genetic professionals, many primary care specialists may actually come to feel quite comfortable managing all genetic patients, except for those with the most complex or esoteric diagnoses.

Clinical genetic services, involving an inherently heavy cognitive component and pre- and postvisit research, require substantial time per patient and are largely inadequately reimbursed by third-party payers. As a result, geneticists are much more likely to practice in a medical school environment and to seek funding from outside sources. In the recent past, many clinical genetic services were supported by collections from clinical laboratory services; however, this source of income is currently being threatened by contracts between insurance carriers and large commercial laboratories that require samples to be sent to one specified laboratory.

With waning clinical laboratory income, geneticists need to find other sources of funding, including additional grant support. Clinical geneticists who teach, oversee a clinical laboratory, and/or do research are more viable overall than those who primarily provide clinical services. Possible exceptions might include geneticists in highly specialized clinical programs, such as grant- or state-supported metabolic centers or multidisciplinary clinics caring for individuals with developmental dis- abilities. Medical geneticists who also see nongenetic patients may have a survival advantage in this ever-changing environment, as well as those geneticists who are trained in a procedure-oriented specialty, such as obstetrics or maternal-fetal medicine. With decreasing numbers of clinical samples coming to small hospital-based laboratories and the failure of geneticists to secure adequate reimbursement for clinical services, genetic units need to find new ways to remain financially solvent while continuing to provide optimal patient care.

With a weekly average of 3.1 hours for seven new patients and 1.7 hours for six follow-up patients, it is clear that genetic practice requires a greater time commitment per patient than other specialties. This time is significantly less than the 7.1 hours (new) and 4.0 hours (follow-up) reported by the clinical genetic group in 1987, but is still far greater than the time spent by most pediatric subspecialists. ${ }^{1}$ Because of the nature of genetic diseases, additional time must often be spent researching rare disorders that are part of the differential diagnosis and explaining complex genetic concepts to parents and patients. If molecular testing is under consideration, additional time must be spent obtaining informed consent from patients before sending out laboratory samples. Furthermore, unlike most specialty and subspecialty practices, geneticists and genetic counselors usually send a written summary of the discussion and findings not only to referring physicians but also to patients and/or families.

The data suggest, however, there may be some flexibility in the practice responsibilities and other professional activities of geneticists. Despite the time-intensive care provided by genetic specialists, two thirds of respondents believed they could reasonably see more patients than they are currently seeing. It is possible that easy access to online resources, division of labor between geneticists and counselors, or the decision by some practitioners to discontinue separate summary letters for parents or patients is decreasing total patient evaluation time. How this relates to reimbursement or compensation was not addressed by the FOPE II survey.

The issues raised by the FOPE II data forces us to ask a rather difficult workforce question. Is there a pressing need to train more clinical geneticists who can provide comprehensive genetic care, or alternatively, is there a need for geneticists to educate primary care providers to incorporate genetics into daily practice? The obvious conflict between the roles of the geneticist as an educator and as a diagnostician and specialist will no doubt be played out in the future in many arenas. As genetic technology moves forward and primary care providers respond to such issues, geneticists will be forced to define more clearly their roles in the overall health care scheme. Because geneticists depend almost entirely on patient referrals from colleagues, the primary care physicians' perceptions about genetics will surely be a driving force that will influence the future direction of this specialty.

As is true with any methodological approach, there are both strengths and potential limitations to the data obtained through the FOPE II survey. The data presented here reflect solely the supply-side perspective of the specialists themselves 
regarding the degree to which they and their colleagues are meeting the current marketplace demand. Despite this limitation, these data represent the first comprehensive study examining the characteristics of practicing geneticists. To address issues such as the adequacy of this specialist workforce more fully, additional studies should be undertaken from other vantage points. For example, household surveys or surveys of health plan members could be conducted to elicit information from a consumer perspective on the availability of, access to, and satisfaction with clinical genetics services. Equally valuable perspectives on these questions could come from surveys of health plan administrators, teaching hospital executives, members of the Association of Medical School Pediatric Department Chairs, fellowship training program directors, and primary care physicians. These stakeholders may have very discrepant views about genetics, as a relatively new and often poorly understood specialty.

The FOPE II survey data themselves cannot suggest potential solutions to the problems currently facing practicing clinical geneticists. However, they provide a starting point for a more global discussion of some pressing practice and public policy issues. It is clear that geneticists must rethink the way they are providing care to address the problems revealed by the survey data and to meet the needs of patients and providers in the future. The following discussion will identify strategies and opportunities for clinical geneticists to (1) increase clinical referrals and patient volume, (2) increase efficiency in the provision of care, and (3) increase revenues to support genetic services.

To increase patient volume, geneticists need to educate health care stakeholders about the types of patients, conditions, and circumstances that require referral for genetic services. Primary care providers, specialist physicians, health care payors, public policy makers, and other stakeholders may not recognize the potential medical and financial benefits of making an initial genetic diagnosis. Such a diagnosis could reduce referrals to multiple medical specialists and provide a rational, focused, efficient, and cost-effective management plan for complex patients. Until these groups recognize the added value of a genetic consultation, patients will not come for such care until all other medical avenues have been exhausted. Geneticists themselves will need to learn how to negotiate a fair and equitable contract for genetic services and to facilitate patient access to genetic care.

As geneticists may begin to see increased patient volume resulting from appropriate referrals, there will need to be a corresponding increase in practice efficiency. Geneticists and genetic counselors, for example, may elect to train genetic assistants to perform some of the more routine genetic counseling for amniocentesis or single gene disorders, such as sickle cell anemia and cystic fibrosis. Although these genetic assistants could see higher volumes of routine patients at a lower cost, they would clearly need to be supervised by a master's level genetic counselor or geneticist, with immediate referral to a counselor and/or physician if unexpected problems were uncovered in the course of counseling. Given growing financial pressures, it may no longer be possible for geneticists and counselors to see patients simultaneously. Patient care may have to be divided into several short sessions, with the counselor initially meeting with the patient to obtain family and medical history information, the physician providing the medical evaluation, and, finally, the genetic counselor or physician alone providing the follow-up plans and diagnostic information. This type of practice efficiency will surely require innovative, logistical, time management planning.

Substantial time savings may also be realized by reducing the amount of paper work generated by each patient visit. In current practice, many geneticists produce an individual letter or clinic note on each patient seen, which is sent to the referring physician and other providers. In the case of many routine patients, however, it may be feasible to develop templates that can be quickly completed for each patient visit. Genetic counselors, moreover, often generate separate letters for their patients that summarize the visit and recommendations in lay language. Use of a standardized template could potentially eliminate the need for such individualized letters. This would also be beneficial for patients, who could leave the office on the day of their visit with a brief summary. For more routine medical issues, genetic practices may choose to create generic fact sheets on common topics that could be given to patients instead of the summary at the time of their appointment.

Additionally, recent advances in information technologies and other electronic media demonstrate significant potential to enhance the efficiency of genetic practice in the future. The electronic medical record, for instance, may permit more timely and efficient transmission of medical information from provider to provider. With newer technologies in voice recognition, moreover, documentation may be accomplished in a matter of a few minutes, rather than a half an hour or more. For genetic practices that require counselors and physicians to travel great distances to see outreach patients, telemedicine may substantially decrease professional time expenditure and allow patients to be seen more quickly, regardless of their geographic location.

Finally, genetic health care providers, many of whom function as an integral part of multidisciplinary health care teams, must ensure that they are adequately and equitably reimbursed for their services. From a financial perspective, the multidisciplinary team approach to clinical care represents a savvy regional marketing draw for patients. This approach also promotes optimal patient care by facilitating the coordinated care of complex patients, who require a wide range of services. Yet, the revenues generated from the care of these patients are generally not pooled for equitable distribution to all team members providing care, including geneticists. To be financially viable providers in the future, geneticists practicing as part of these teams will need to secure equitable compensation for their clinical services. Genetic counselors in hospital settings likewise serve as part of multidisciplinary clinical teams, often providing services without reimbursement to clinical programs in a wide range of specialties. Hospitals and other em- 
ployers should provide salary support for genetic counselors to compensate them for these services.

Beyond their duties as clinicians and researchers, geneticists serve as educators in a variety of settings. Geneticists are generally recognized to be the primary genetic educational resource for residency and fellowship programs. Although each training program must comply with very specific requirements regarding genetic education hours, most institutions have a very limited number of trained genetic professionals to provide these services. As geneticists and genetic counselors are increasingly asked to teach genetics to surgical, medical, and dental trainees, the teachers need to seek adequate and equitable compensation for their educational services. The value of genetic education and consultative services is even more apparent in the fields of pediatrics and obstetrics, with increasing numbers of neonatal admissions involving children with birth defects and/or genetic disorders, as well as enhanced prenatal detection of fetal anomalies.

In addition to geneticists, genetic counselors and other professionals are asked to provide both informal and formal consultative services while participating in teaching conferences and bedside teaching rounds for the benefit of medical students, residents, fellows, and unit staff in pediatrics and other specialties. This work done needs to be recognized and financially supported as a valuable part of these training programs. Until recently, genetic counselors in academic centers, as well as less traditional practice settings, have been unable to bill directly for their clinical services. If more states grant licensure for genetic counselors in the future, these providers should be able to bill health plans directly for their services. This may provide additional income from direct patient care to support genetic service programs.

Like the genetic revolution itself, the specialty of medical genetics is undergoing rapid change. The role of medical geneticists in education, research, and patient care is evolving in a competitive health care environment that may not support many of this specialty's activities, despite a clear societal need to understand and apply genetic information. ${ }^{12}$ More research is needed on the medical genetics workforce and access to genetic services. The interface between primary care physicians and geneticists also needs to be examined more closely. For now, the views expressed by geneticists through the FOPE II data provide a crucial and previously unavailable perspective on their own personal perceptions about the current practice of genetics. However, additional work is needed to obtain a much broader overview of the direction of the specialty and how it fits into the larger health care picture.

\section{Acknowledgments}

This work was supported by grants from the Center for the Future of Children of the David and Lucille Packard Foundation, the Maternal and Child Health Bureau (Project MCJ379381), the Association of Medical School Pediatric Department Chairmen, the American Board of Pediatrics Foundation, and the American Academy of Pediatrics. The authors thank the Project members and staff of the Future of Pediatric Education II (FOPE II) Project; Thomas M. Gorey, JD; the members of the AAP Section on Genetics and Birth Defects; the members of the AAP Committee on Pediatric Workforce Subcommittee on Subspecialty Workforce; and the many other physicians who responded to the survey for their essential contributions to this project.

\section{References}

1. Bernhardt BA, Weiner J, Foster EC, Tumpson JE, Pyeritz RE. The economics of clinical genetic services. II. A time analysis of a medical genetic clinic. Am J Hum Genet 1987;41:559-565.

2. Pyeritz RE, Tumpson JE, Bernhardt BA. The economics of clinical genetic services. I. Preview. Am J Hum Genet 1987;41:549-558.

3. Myers MF, Doksum T, Holtzman NA. Genetic services for common complex disorders: surveys of health maintenance organizations and academic genetic centers. Genet Med 1999;1:248-253.

4. Task Force of the Force of the Future of Pediatric Education II (FOPE II) Project. Organizing pediatric education to meet the needs of infants, children, adolescents and young adults in the 21st century. Pediatrics 2000;105(suppl 2):157-212.

5. Kramer MS. Clinical epidemiology and statistic: a primer for clinical investigators and decision-makers. Berlin, New York: Springer-Verlag, 1998.

6. Cooper-Patrick L, Gallo JJ, Gonzales JJ, Vu HT, Powe NR, Nelson C, Ford DE. Race, gender, and partnership in the patient physician relationship. JAMA 1999;282:583589.

7. Flores G. Culture and the patient-physician relationship: achieving cultural competency in health care. J Pediatr 2000;136:14-23.

8. Saha S, Taggart SH, Komaromy M, Bindman AB. Do patients choose physicians of their own race? Health Aff 2000;19:76-83.

9. Weinick RM, Krauss NA. Racial/ethnic differences in children's access to care. Am J Public Health 2000;90:1771-1774.

10. Stoddard JJ, Cull WL, Jewett EAB, Brotherton SE, Mulvey HJ, Alden ER. Providing pediatric subspecialty care: a workforce analysis. Pediatrics 2000;106:1325-1333.

11. ACP-ASIM Observer. Genetic counseling: your new job. Are you ready to handle public misconceptions, confidentiality dilemmas? February 1995.

12. Kinmouth AL, Reinhard J, Bobrow M, Pauker S. The new genetics: implications for clinical services in Britain and the United States. BMJ 1998;316:767-770. 